\title{
Streptococcus equi subsp. zooepidemicus
}

National Cancer Institute

\section{Source}

National Cancer Institute. Streptococcus equi subsp. zooepidemicus. NCI Thesaurus.

Code $C 124408$.

A subspecies of Streptococcus equi that is positive for beta hemolysis, produces

hyaluronic acid but not streptolysin $\mathrm{O}$, and occurs in pairs or long chains. This subspecies is also lactose positive and capable of fermenting sorbitol but not trehalose. 